\title{
Using Paraffin PCM for Thermal Management of BOLAS Planetary CubeSats with Ion Thrusters
}

\author{
Michael K. Choi* \\ NASA Goddard Space Flight Center, Greenbelt, MD 20771
}

\begin{abstract}
The Bi-sat Observations of the Lunar Atmosphere above Swirls (BOLAS) is a NASA planetary CubeSat mission concept in low lunar orbit. The BOLAS lower CubeSat is at a 90 $\mathbf{k m}$ altitude above the lunar surface during spiraling down from the Evolved Expendable Launch Vehicle (EELV) Secondary Payload Adapter (ESPA) to the Moon. Without phase change material (PCM), the worst hot case temperature prediction for the Command and Data Handling (C\&DH) exceeds the $61^{\circ} \mathrm{C}$ maximum operating limit, and those for the Iris solid state power amplifier (SSPA) and transponder exceed the $50^{\circ} \mathrm{C}$ maximum operating limit. Miniature n-Tricosane PCM packs on the Iris SSPA and transponder, and miniature n-Hexacosane PCM packs on the C\&DH are used to store thermal energy in sunlight and release it in the eclipse. With paraffin PCM, all the temperatures are within the maximum operating limits.
\end{abstract}

\section{Nomenclature}

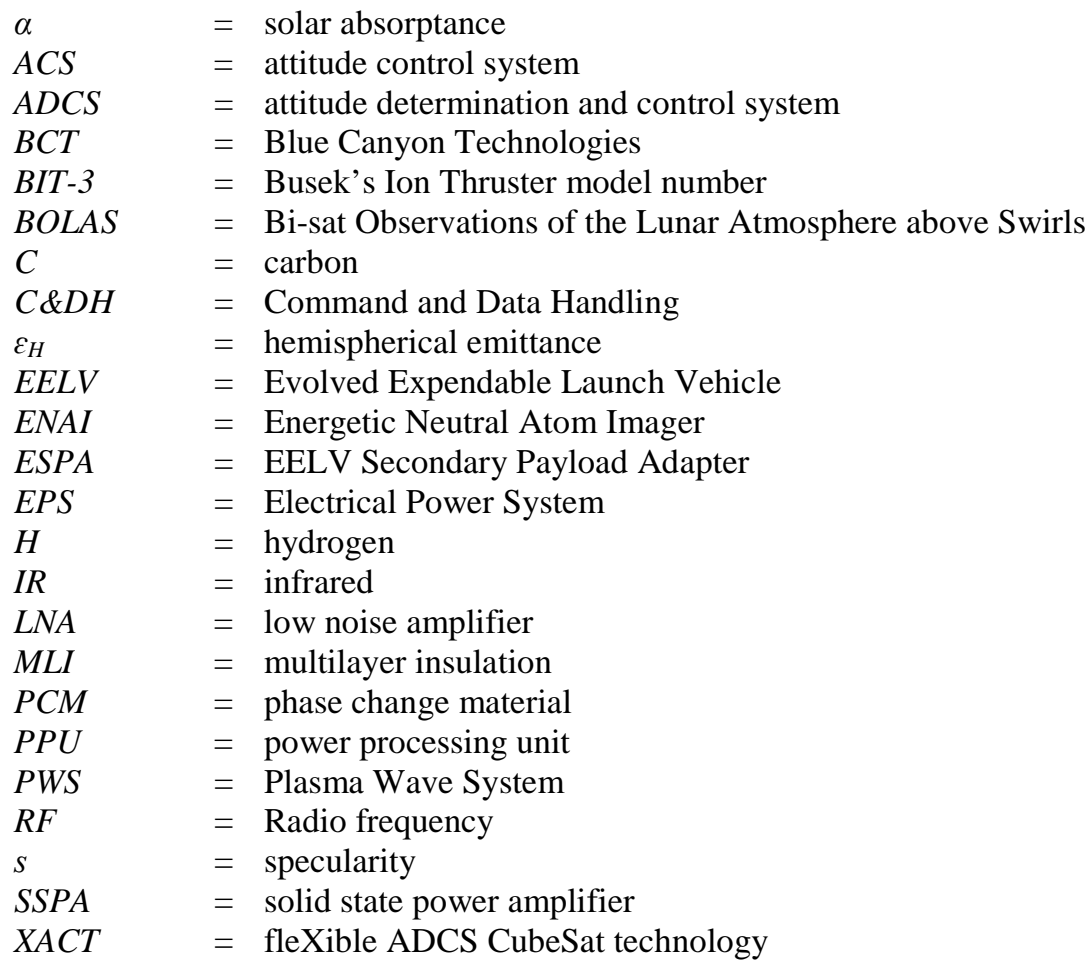

\section{Introduction}

BOLAS is a NASA's tethered planetary CubeSat mission concept. ${ }^{1}$ Two CubeSats (lower and upper) are connected by a thin, miles-long tether. After BOLAS is deployed from the C\&DEELV ESPA, electrical propulsion, which includes two Busek's ion thrusters (model BIT-3), thrusts it to the Moon. Once captured at Moon, the BIT-3s

\footnotetext{
* Senior Aerospace Engineer, Heat Transfer, AIAA Associate Fellow.
} 
thrust BOLAS down to frozen science orbit. From the frozen orbit, a $25 \mathrm{~km}$ long tether deploys and the BOLAS CubeSat's separate. The instruments operate most of the time once the tether is deployed, and minimal BIT-3 thrusting is anticipated. The articulated solar array tracks the sun after it is deployed.

Figures 1 and 2 show the BOLAS lower and upper CubeSat respectively. They are virtually identical, except that the upper CubeSat does not have the Energetic Neutral Atom Imager (ENAI) instrument. Figure 3 shows the layout of the lower CubeSat components. Tables 1 and 2 present the power dissipation of the spacecraft and payload respectively on each BOLAS CubeSat. The BIT-3 and payload are not powered on simultaneously. Table 3 compares the power dissipation of each BOLAS CubeSat during the science mode and propulsion mode. During the science mode, the Lower CubeSat and Upper CubeSat have 99.3 W and 97.3 W power dissipation respectively. During the propulsion mode, each CubeSat has a $159.8 \mathrm{~W}$ power dissipation. Table 4 presents the allowable flight temperature limits for the BOLAS CubeSat's.

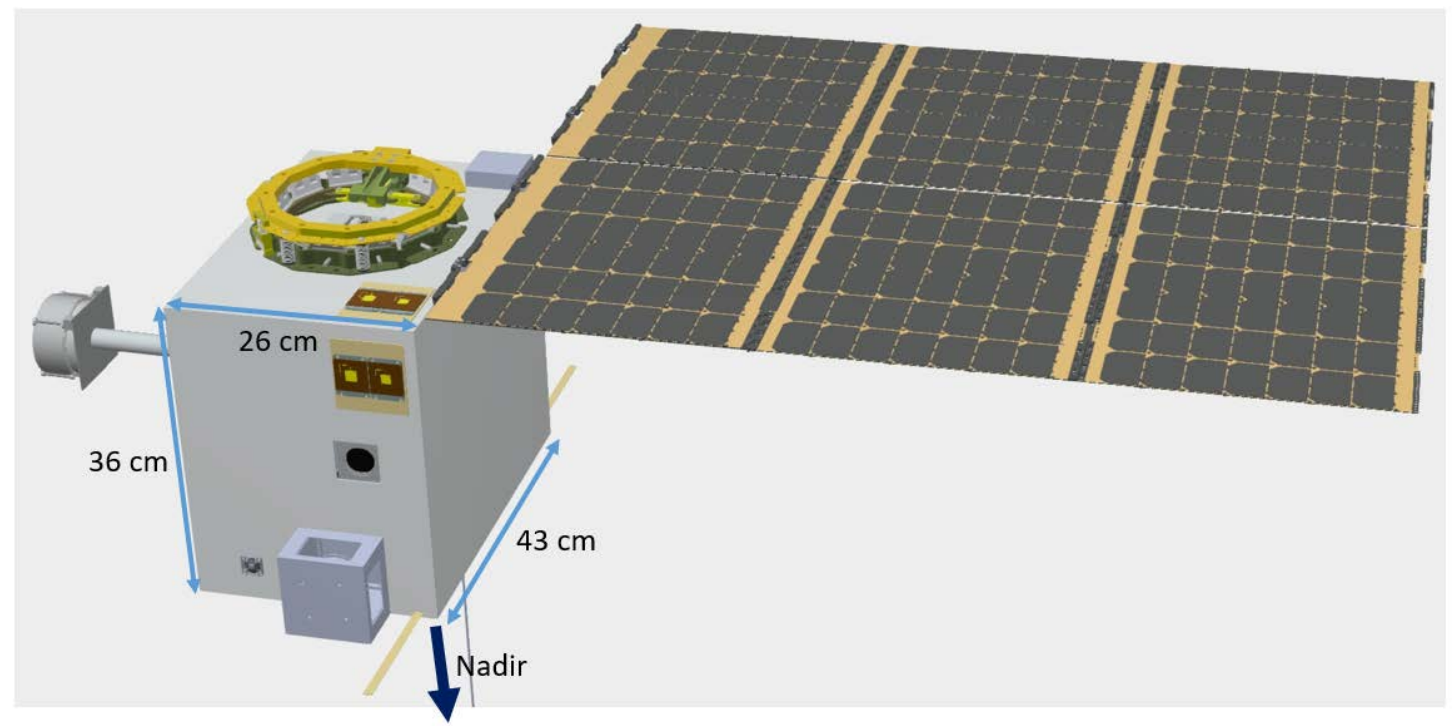

Fig. 1. BOLAS lower spacecraft.

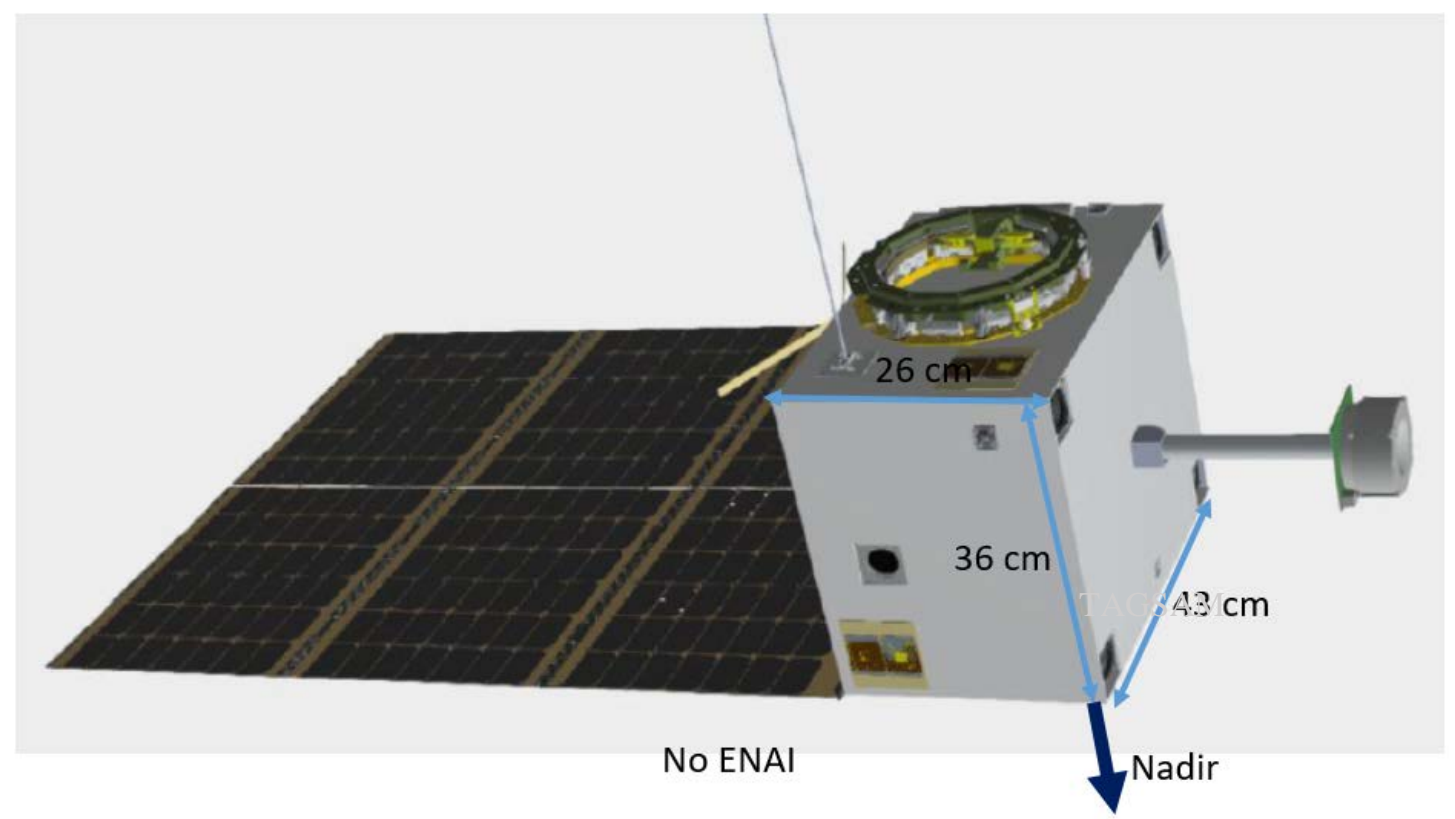

Fig. 2. BOLAS upper spacecraft. 


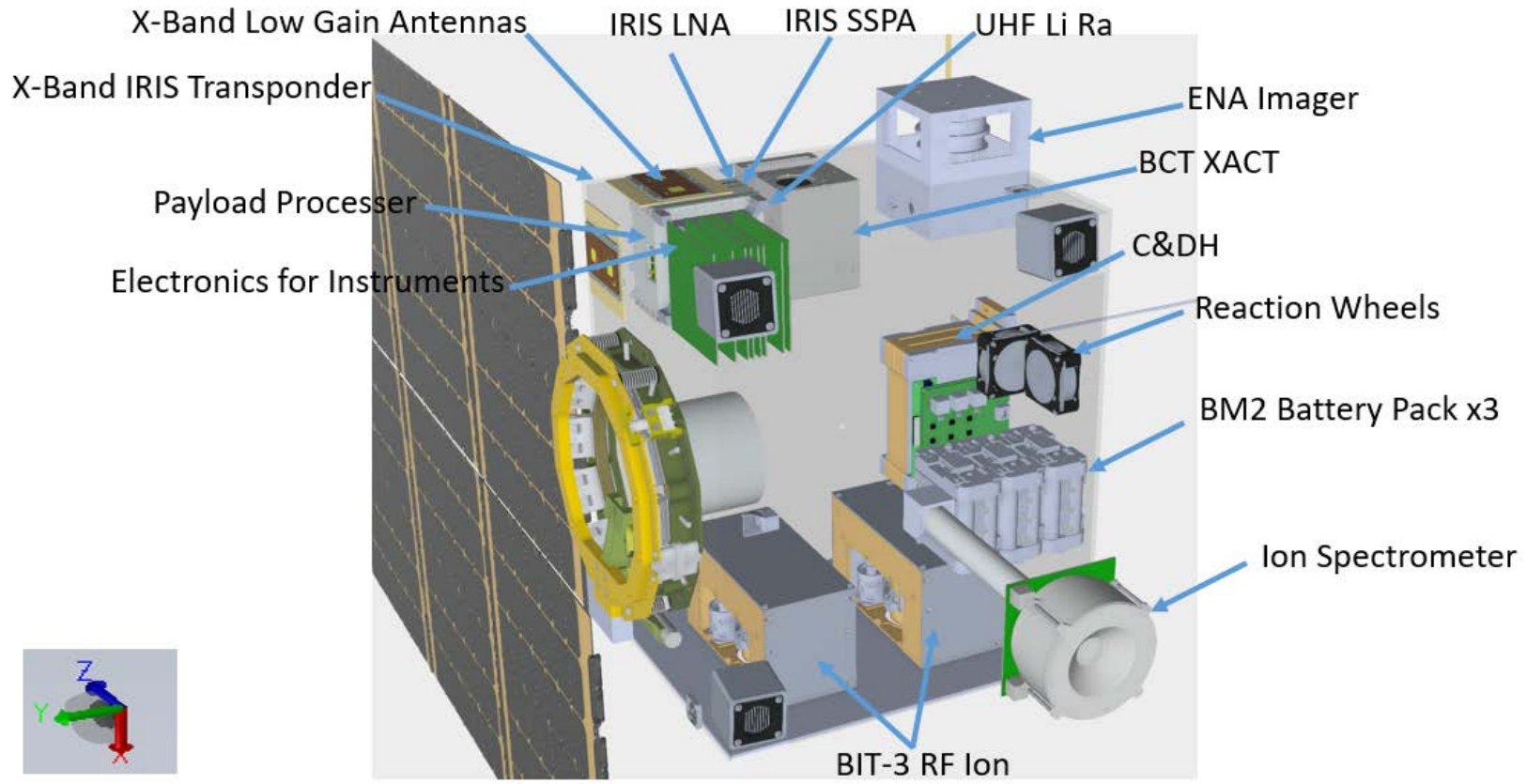

Fig. 3. Lower spacecraft and payload components.

Table 1. Spacecraft Power Dissipation.

\begin{tabular}{|c|c|c|}
\hline & & Power Dissipation (W) \\
\hline C\&DH & Proton $400 \mathrm{~K}$ & 13.3 \\
\hline \multirow[t]{3}{*}{ Electrical Power } & $\begin{array}{l}\text { Articulated Solar Array (Solar } \\
\text { panels, gimbals \& drive } \\
\text { articulators) }\end{array}$ & 5.56 \\
\hline & EPS & 2.22 \\
\hline & Batteries & \\
\hline AD\&ACS & $\begin{array}{c}\text { BCT XACT (Star Tracker, Reaction } \\
\text { Wheels, Sun Sensor) }\end{array}$ & $\begin{array}{c}2.78 \text { (Star Tracker + Sun Sensor) } \\
20 \text { (Reaction Wheels) }\end{array}$ \\
\hline ACS & ACS Thrusters + PPU & $\begin{array}{l}5.5 \text { (assumes two thrusters } \\
\text { operating simultaneously) }\end{array}$ \\
\hline \multirow[t]{3}{*}{ Communications } & JPL Iris LNA & 1 \\
\hline & Iris SSPA & 27 \\
\hline & Iris Transponder & 12.44 \\
\hline Propulsion & BIT-3 RF Ion (2) & 35 Each \\
\hline $\begin{array}{l}\text { Tether and Deployment System } \\
\text { (Upper CubeSat Only) }\end{array}$ & Deployment System & $\begin{array}{l}4.8 \text { (pinch roller motor) } \\
5.0 \text { (motor controller) }\end{array}$ \\
\hline
\end{tabular}


Table 2. Payload Power Dissipation.

\begin{tabular}{|c|c|c|}
\hline & & Power Dissipation (W) \\
\hline Payload Processor & DM & 6 Average \\
\hline Payloads & $\begin{array}{c}\text { Energetic Neutral Atom Imager } \\
\text { (ENAI) (Lower CubeSat Only) }\end{array}$ & $1.5-2.5$ \\
\cline { 2 - 3 } & Plasma Wave System (PWS) & 2 \\
\cline { 2 - 3 } & Ion Spectrometer & $1.5-2.5$ \\
\cline { 2 - 3 } & Magnetometer Sensor & $<1$ (Sensor + Electronics) \\
\cline { 2 - 3 } & Magnetometer Electronics & $<1$ \\
\cline { 2 - 3 } & Cameras (secondary instruments) & \\
\hline
\end{tabular}

Table 3. Science Mode versus Propulsion Mode Power Dissipation.

\begin{tabular}{|c|c|c|}
\hline Component & Electric Propulsion On (W) & Electric Propulsion Off (W) \\
\hline BIT-3 Thruster and PPU & 75.5 & 0 \\
\hline Spacecraft & 84.3 & 84.3 \\
\hline Payload & 0 During Propulsion Mode & $\begin{array}{c}18 \text { (Lower CubeSat ); } 16 \text { (Upper } \\
\text { CubeSat ) } \\
\text { During Science Mode }\end{array}$ \\
\hline Total & 159.8 During Propulsion Mode & $\begin{array}{c}99.3 \text { (Lower CubeSat ); } 97.3 \\
\text { (Upper CubeSat ) } \\
\text { During Science Mode }\end{array}$ \\
\hline
\end{tabular}


Table 4. Allowable Flight Temperature Limits.

\begin{tabular}{|c|c|c|c|}
\hline & & Operating $\left({ }^{\circ} \mathrm{C}\right)$ & $\begin{array}{l}\text { Non-Operating } \\
\left({ }^{\circ} \mathrm{C}\right)\end{array}$ \\
\hline \multicolumn{4}{|c|}{ Spacecraft Subsystems } \\
\hline $\mathrm{C} \& \mathrm{DH}$ & Proton $400 \mathrm{~K}$ & -24 to +61 & -40 to +85 \\
\hline \multirow[t]{3}{*}{ Electrical Power } & $\begin{array}{c}\text { Solar Array Gimbals \& Drive } \\
\text { Articulators }\end{array}$ & -30 to +70 & -30 to +70 \\
\hline & EPS & -40 to +85 & \\
\hline & Batteries & $\begin{array}{c}\text { Charge: } 0 \text { to }+40 \\
\text { Discharge: }-20 \text { to }+60\end{array}$ & -20 to +40 \\
\hline AD\&ACS & $\begin{array}{c}\text { BCT XACT (Star Tracker, } \\
\text { Reaction Wheels, Sun Sensor) }\end{array}$ & $\begin{array}{c}+10 \text { to }+30 \text { (Star Tracker) } \\
-30 \text { to }+70 \text { (Reaction } \\
\text { Wheels) }\end{array}$ & -30 to +70 \\
\hline ACS & ACS Thrusters + PPU & -20 to +70 & \\
\hline \multirow[t]{3}{*}{ Communications } & JPL Iris LNA & -20 to +50 & -20 to +50 \\
\hline & Iris SSPA & -20 to +50 & -20 to +50 \\
\hline & Iris Transponder & -20 to +50 & -20 to +50 \\
\hline Propulsion & BIT-3 RF Ion (2) & -20 to +70 & -30 to +80 \\
\hline $\begin{array}{l}\text { Tether and Deployment } \\
\text { System }\end{array}$ & Deployment System & $<70$ & \\
\hline \multicolumn{4}{|c|}{ Payload Subsystems } \\
\hline Payload Processor & DM & 0 to +75 & -10 to +80 \\
\hline \multirow[t]{6}{*}{ Payloads } & $\begin{array}{l}\text { Energetic Neutral Atom Imager } \\
\text { (ENAI) }\end{array}$ & -30 to +50 & -35 to +60 \\
\hline & Plasma Wave System (PWS) & -50 to +50 & \\
\hline & Ion Spectrometer & -30 to +50 & -35 to +60 \\
\hline & Magnetometer Sensor & -55 to +60 & -80 to +85 \\
\hline & Magnetometer Electronics & -30 to +60 & -55 to +85 \\
\hline & Cameras (secondary instruments) & -10 to +55 & -25 to +70 \\
\hline
\end{tabular}




\section{Thermal Environment}

The BOLAS temperatures are highly dependent on its altitude above the Moon's surface. Figure 4 shows the infrared (IR) flux map of the Moon. ${ }^{2}$ It varies from 1,335 $\mathrm{Wm}^{-2}$ maximum on the sun side to $5 \mathrm{Wm}^{-2}$ minimum on the dark side. Table 5 presents the solar irradiance, albedo factor, and IR flux in the hot case and cold case. The BOLAS Bi-sat requires its thermal system to accommodate excursions due to elliptical frozen lunar orbits with a $30^{\circ}$ inclination with periapsis altitudes ranging from 14.51 to $59.7 \mathrm{~km}$. The lower altitude CubeSat has a closest approach to the Moon of $2.01 \mathrm{~km}$ at periapsis. Additionally, after the joined Bi-sat is deployed from the propulsive EELV Secondary Payload Adapter (ESPA) at first Lagrange point (L1), spiraling down from a high-altitude $30^{\circ}$ inclination lunar orbit to the frozen lunar orbit adds a different thermal environment because the two CubeSat's view each other prior to separation. Emphasis of the BOLAS thermal analysis is placed on the hot case to assure the temperatures are within the maximum operating allowable flight temperature limits.

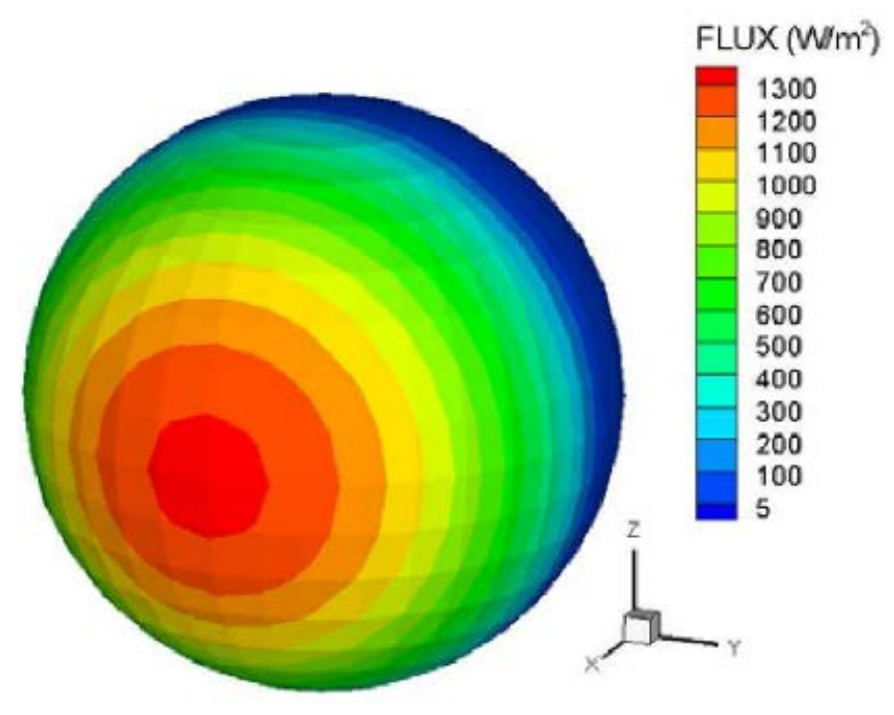

Fig. 4. IR flux map of the Moon for thermal analysis.

Table 5. Environmental Fluxes at Moon for Thermal Analysis.

\begin{tabular}{|c|c|c|}
\hline & Hot Case & Cold Case \\
\hline Solar Irradiance $\left(\mathrm{Wm}^{-2}\right)$ & 1420 & 1280 \\
\hline Albedo Factor & 0.13 & 0.06 \\
\hline IR on Sun Side $\left(\mathrm{Wm}^{-2}\right)$ & 1335 Peak & 1114 Peak \\
\hline IR on Dark Side $\left(\mathrm{Wm}^{-2}\right)$ & 5 & 5 \\
\hline
\end{tabular}

\section{Thermal Management Approach}

The BOLAS Bi-sat is exposed to different thermal environment after it is deployed from the ESPA at L1, due to changes in altitudes above the Moon's surface. Its power dissipation is highly dependent on the mode of operation. During spiraling down from L1 to the frozen lunar orbit, the BIT-3s are powered on and thrust continuously, and the instruments are powered off. In the frozen lunar orbits, the instruments are powered on and the BIT-3s are powered off. During frozen orbit altitude changes, the BIT-3s are powered on for thrusting and the instruments are powered off. The power dissipation of each CubeSat is $159.8 \mathrm{~W}$ maximum when the instruments are powered off and BIT-3s are powered on. Its minimum is $99.3 \mathrm{~W}$ for the lower altitude CubeSat or $97.3 \mathrm{~W}$ for the upper altitude CubeSat when the BIT-3s are powered off and the instruments are powered on. As a result, when the 
BIT-3s are powered on for thrusting, the waste heat for thermal management is over $60 \mathrm{~W}$ larger than that of the science operation mode. Each CubeSat has an articulated solar array to track the sun. It has a thermal effect on the chassis. The BOLAS Bi-sat is cooled passively or radiatively. The entire exterior of the lower altitude or upper altitude CubeSat chassis and backside of the solar array are painted with the Z93C55 white paint, which has a low absorptance and a high emittance. It is conductive to meet the electrostatic discharge (ESD) requirement. It has been flown on multiple NASA missions. The power dissipation of components is transferred to the chassis by conduction through the mounting interfaces with thermal interface materials. There are good conduction paths between adjacent equipment panels of the chassis. The Ion Spectrometer (IS) boom is thermally isolated from the chassis or structure by G10 spacers. The exterior of the IS and its boom is also painted with Z93C55 white paint. In order to meet the star tracker thermal requirement, it is thermally isolated from the chassis and cooled passively by a small radiator plate located at the IS side of the chassis. G10 spacers and multilayer insulation (MLI) blanket are used for thermal isolation. The star tracker radiator is coated with the Z93C55 white paint and is thermally isolated from the chassis by G10 spacers. A Mylar blanket encapsulated Pyrovo pyrolytic graphite film (PGF) flexible thermal strap transfers heat from the star tracker to the radiator. Miniature constant conductance heat pipes of $3.175 \mathrm{~mm}$ outer diameter spread heat from the Iris SSPA and transponder to three other sides of the chassis that have low or no power dissipation. The exterior of components internal to the chassis has a high emittance to enhance heat radiation. Additionally, miniature paraffin phase change material (PCM) packs are used to store thermal energy in sunlight and release it in the eclipse to prevent the temperatures of the Iris SSPA, transponder, and C\&DH from exceeding the maximum operating allowable flight temperature limits. The PCM on the Iris SSPA and transponder is n-Tricosane $\left(\mathrm{C}_{23} \mathrm{H}_{48}\right)$, which has a $47^{\circ} \mathrm{C}$ melting point and $170 \mathrm{~kJ} / \mathrm{kg}$ enthalpy of fusion. The mass of miniature n-Tricosane PCM packs, including the aluminum structure, is $220 \mathrm{~g}$ per CubeSat. The PCM on the C\&DH is n-Hexacosane $\left(\mathrm{C}_{27} \mathrm{H}_{56}\right)$, which has a $59^{\circ} \mathrm{C}$ melting point and $172.6 \mathrm{~kJ} / \mathrm{kg}$ enthalpy of fusion. The mass of miniature n-Hexacosane PCM packs, including the aluminum structure, is $50 \mathrm{~g}$ per CubeSat.

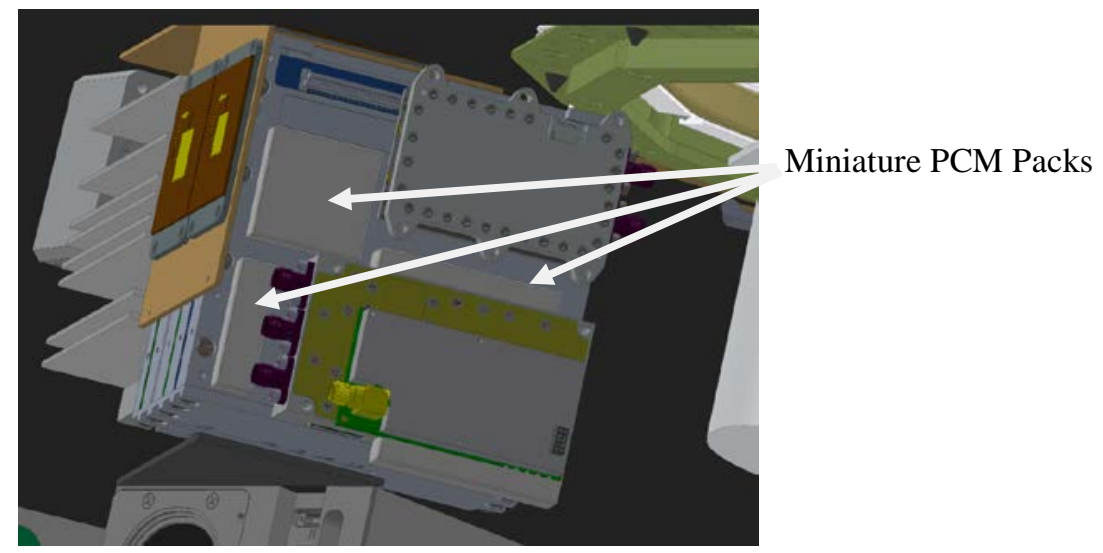

Fig. 5. Miniature PCM packs on SSPA and transponder.

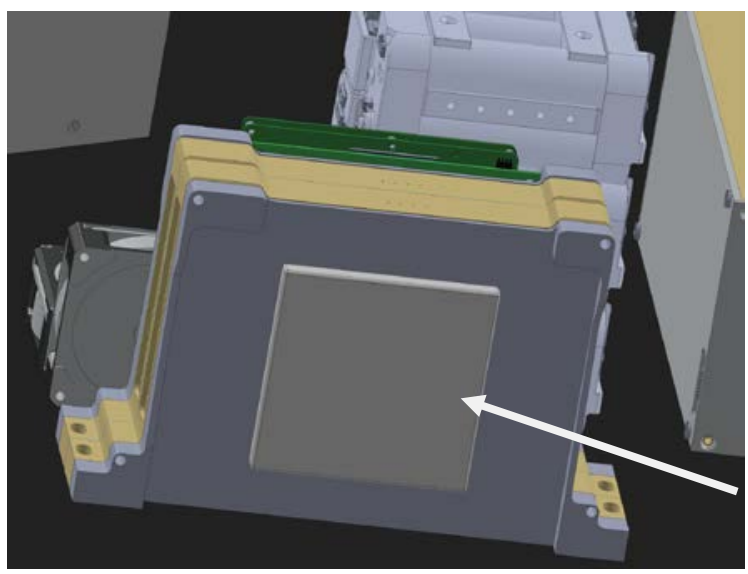

Miniature PCM Pack

Fig. 6. Miniature PCM pack on C\&DH.

American Institute of Aeronautics and Astronautics 


\section{Thermal Model and Predictions}

This section presents the BOLAS thermal modeling, analysis, and temperature predictions.

\section{A. Thermal Model}

The BOLAS thermal model is in Autodesk AutoCAD 2018 Thermal Desktop ${ }^{\mathrm{TM}}$ version 5.8 format. Figure 7 shows the thermal model of the Bi-sat after it is deployed from the ESPA at L1 and prior to separation at the Moon.

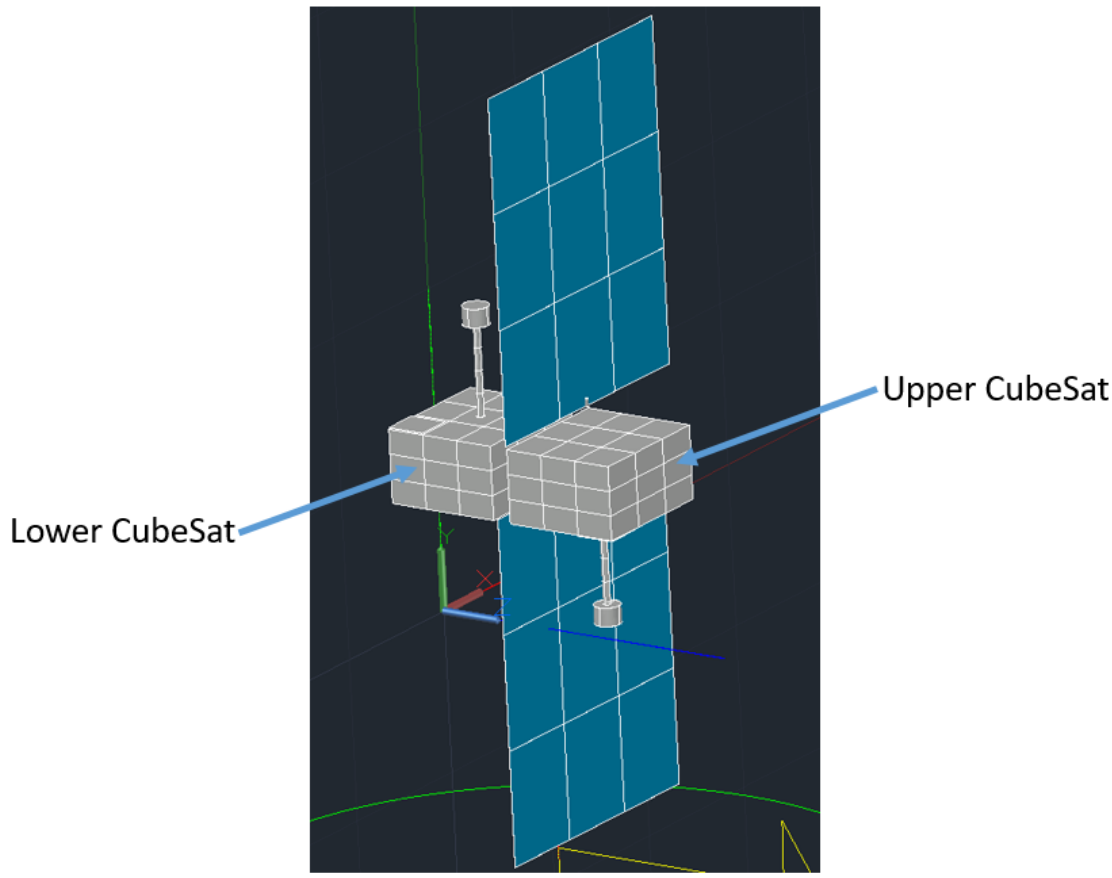

Fig. 7. Thermal model of bi-sat prior to separation.

Figure 8 shows the Bi-sat in a 1,000 km altitude above the lunar surface during spiraling down from the ESPA to the moon.

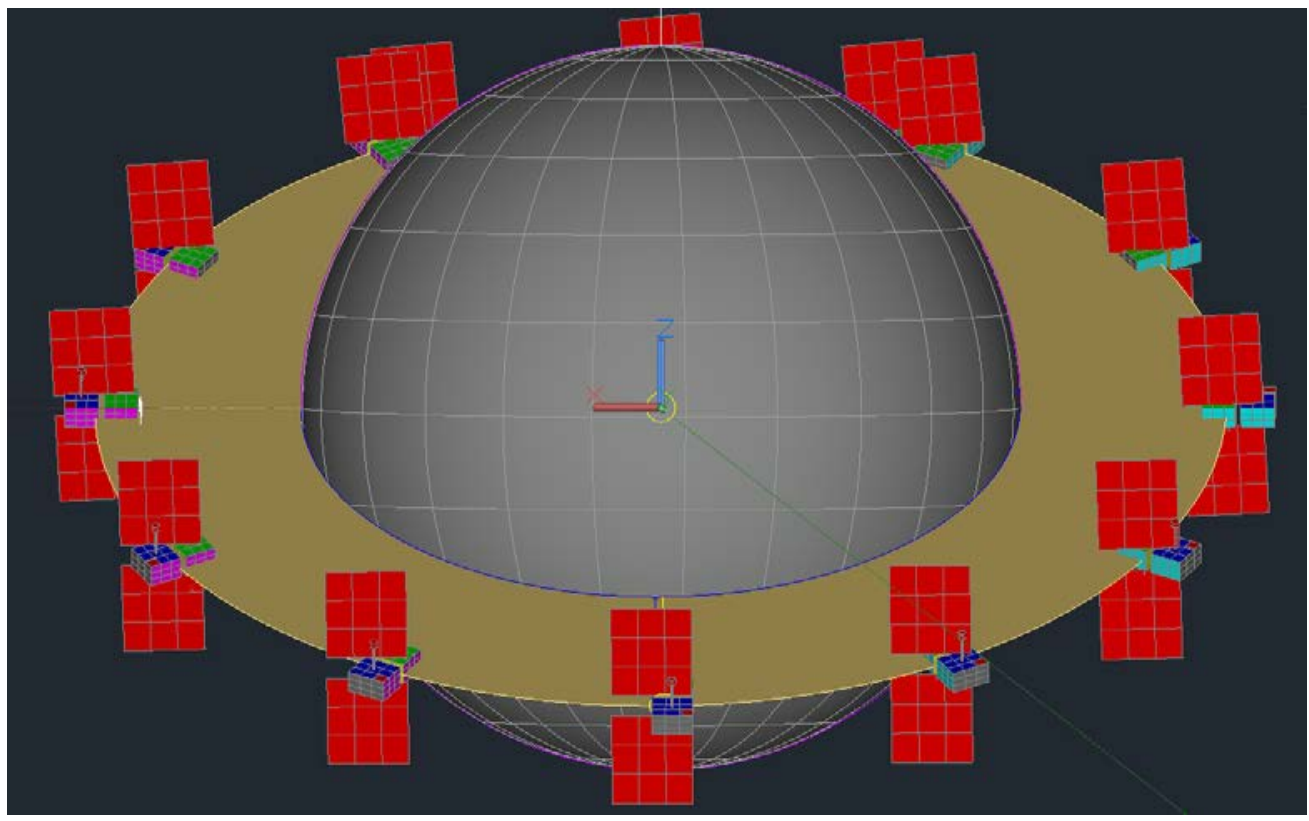

Fig. 8. Thermal model of bi-sat at $1,000 \mathrm{~km}$ altitude during spiraling down from ESPA. 
Figure 9 shows the lower altitude CubeSat after its separation from the upper altitude CubeSat.

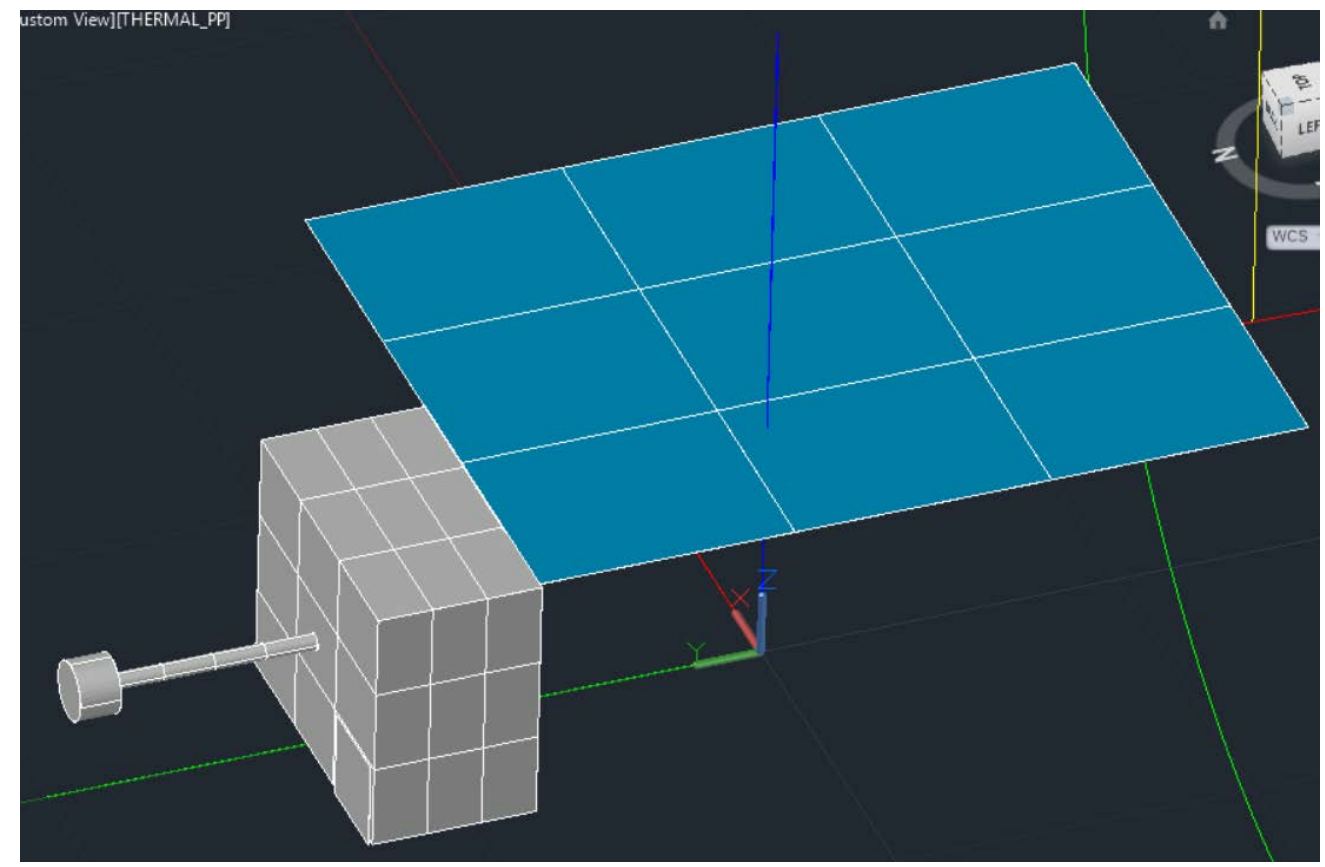

Fig. 9. Thermal model of lower CubeSat after separation from upper CubeSat.

Figure 10 shows the lower altitude CubeSat in an elliptical orbit with a $2.01 \mathrm{~km}$ true perigee altitude and an eccentricity of 0.039184 .

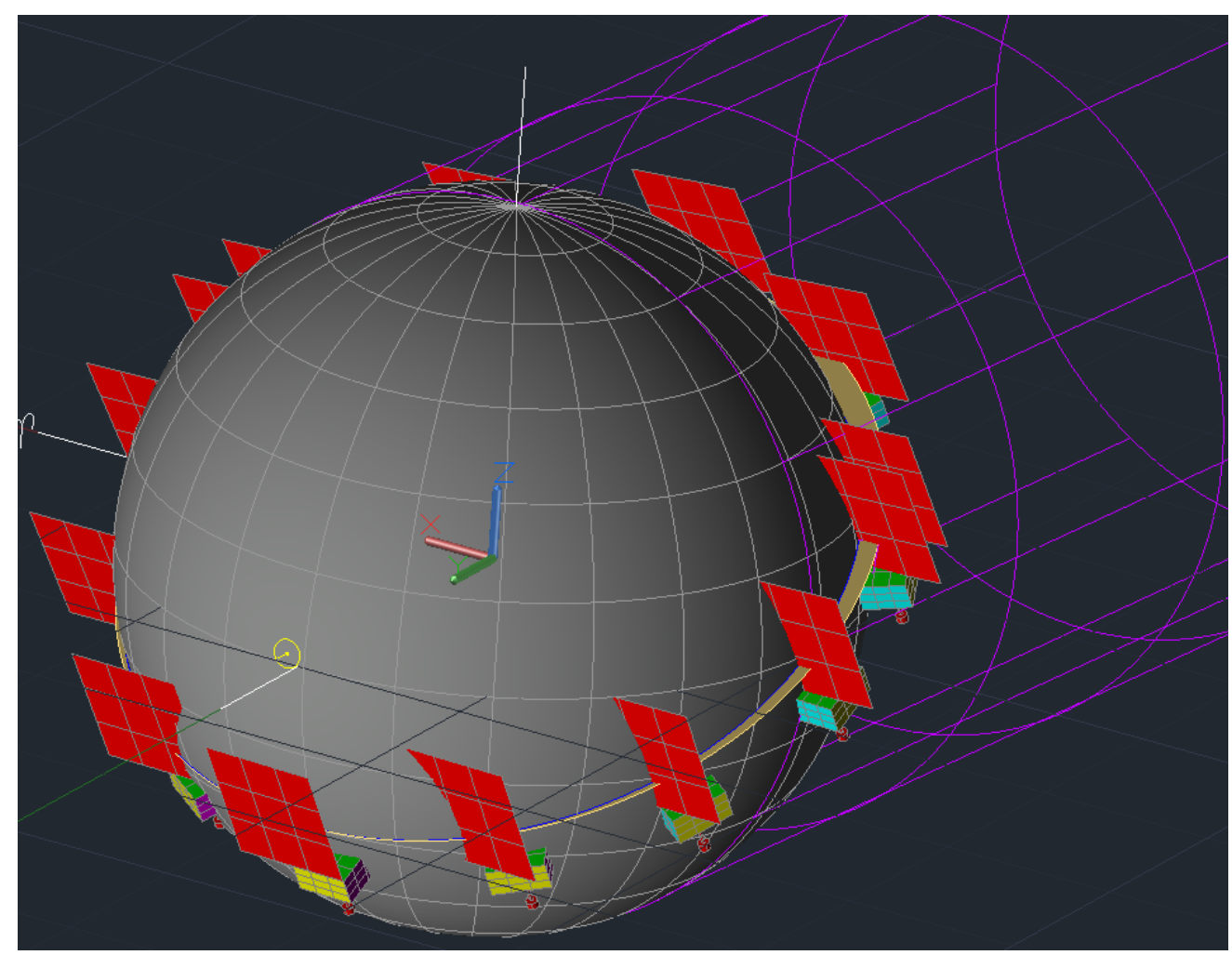

Fig. 10. Thermal model of lower CubeSat at $2.01 \mathrm{~km}$ perigee altitude and 0.039184 eccentricity orbit. 


\section{B. Thermo-optical Properties}

Table 6 presents the solar absorptance $(\alpha)$, hemispherical emittance $\left(\varepsilon_{\mathrm{H}}\right)$ and specularity (s) used in the thermal model. Emphasis of the BOLAS thermal design is placed on the end of life hot case to prevent component temperatures from exceeding the maximum operating allowable flight temperature limits.

Table 6. Thermo-optical Properties.

\begin{tabular}{|c|c|c|c|c|}
\hline Description & \multicolumn{4}{|c|}{ Beginning of Life Cold Case } \\
\hline Coatings & $\alpha$ & $\varepsilon_{\mathrm{H}}$ & $\mathrm{s}$ (Solar) & $\mathrm{s}$ (IR) \\
\hline Solar Array Cells & 0.89 & 0.88 & 0.80 & 0.80 \\
\hline Z93C55 White Paint & 0.12 & 0.91 & 0.05 & 0.05 \\
\hline
\end{tabular}

\begin{tabular}{|c|c|c|c|c|}
\hline Description & \multicolumn{4}{|c|}{ End of Life Hot Case } \\
\hline Coatings & $\alpha$ & $\varepsilon_{\mathrm{H}}$ & $\mathrm{s}$ (Solar) & $\mathrm{s}$ (IR) \\
\hline Solar Array Cells & 0.90 & 0.84 & 0.90 & 0.90 \\
\hline Z93C55 White Paint & 0.20 & 0.86 & 0.05 & 0.05 \\
\hline
\end{tabular}

\section{Thermal Predictions}

The worst hot case maximum solar array temperature prediction is $113^{\circ} \mathrm{C}$. Gerhardt, D., et al. reported the flight temperature of the Colorado Student Space Weather Experiment (CSSWE) body mounted solar arrays in low Earth orbit was up to $120^{\circ} \mathrm{C}$ when its spin rate was close to zero. ${ }^{3}$ Figure 11 shows the relationship between the solar array voltage and temperature. ${ }^{4}$ As the solar array temperature increases, the voltage decreases. The BOLAS solar array area is adequate and no electrical power issue is expected, despite the maximum temperature prediction is $113^{\circ} \mathrm{C}$.

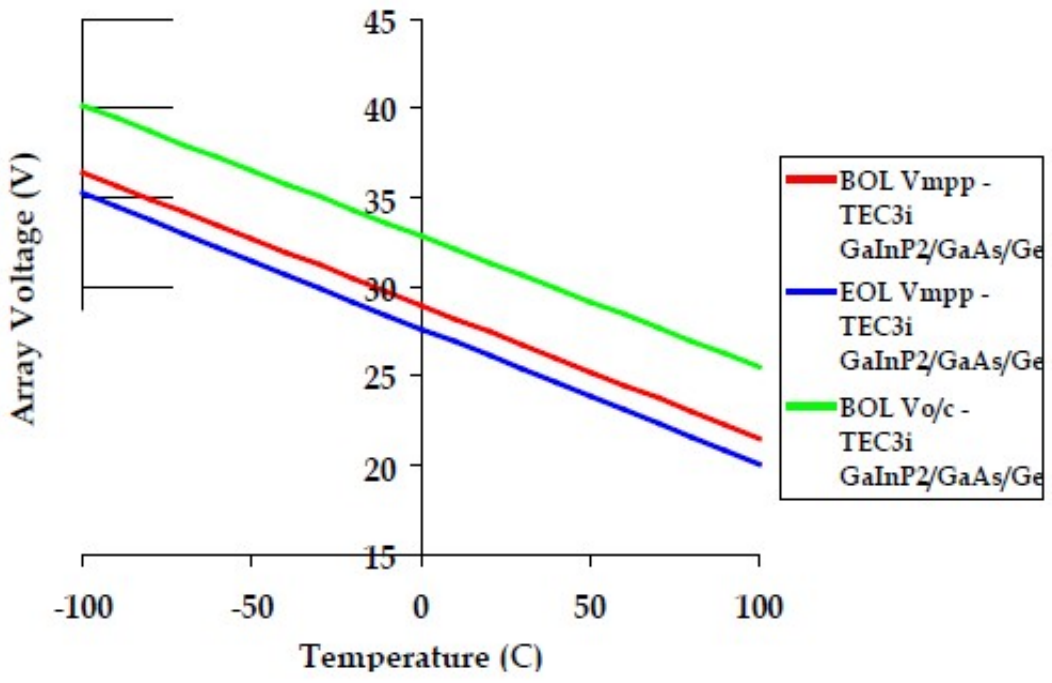

Fig. 11. Solar array voltage versus temperature. ${ }^{4}$

During spiraling down from L1 to the frozen lunar orbit, the thermal effect of lunar flux on the BOLAS Bi-sat increases as the altitude above the lunar surface decreases. Figure 12 presents the worst hot case temperature predictions for the BOLAS Bi-sat in a lunar orbit of 10,000 km altitude above the lunar surface during spiraling 
down from the ESPA to the Moon. All the temperatures are within the allowable flight temperature limits.
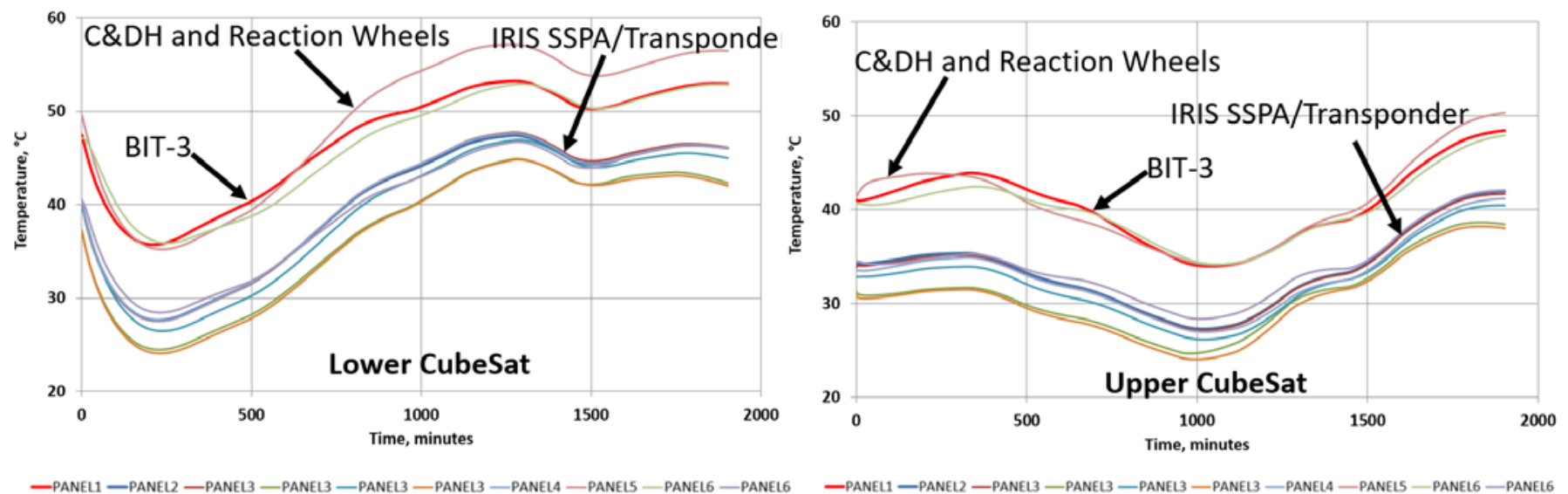

Fig. 12. Hot case temperature predictions in lunar orbit at $10,000 \mathrm{~km}$ altitude above lunar surface during spiraling down from ESPA to Moon.

Figure 13 presents the worst hot case temperature predictions for the BOLAS lower altitude CubeSat in a lunar orbit and at a $90 \mathrm{~km}$ altitude above the lunar surface during spiraling down from the ESPA to the Moon. Without PCM, the C\&DH temperature exceeds the $61^{\circ} \mathrm{C}$ operating maximum allowable flight temperature limit, and the Iris SSPA and transponder temperatures exceed the $50^{\circ} \mathrm{C}$ operating maximum allowable flight temperature limit. With PCM, all the temperatures are within the allowable flight temperature limits. The n-Hexacosane PCM on the C\&DH melts in sunlight and freezes in the eclipse at $59^{\circ} \mathrm{C}$. The n-Tricosane PCM on the Iris SSPA and transponder melts in sunlight and freezes in the eclipse at $47^{\circ} \mathrm{C}$.
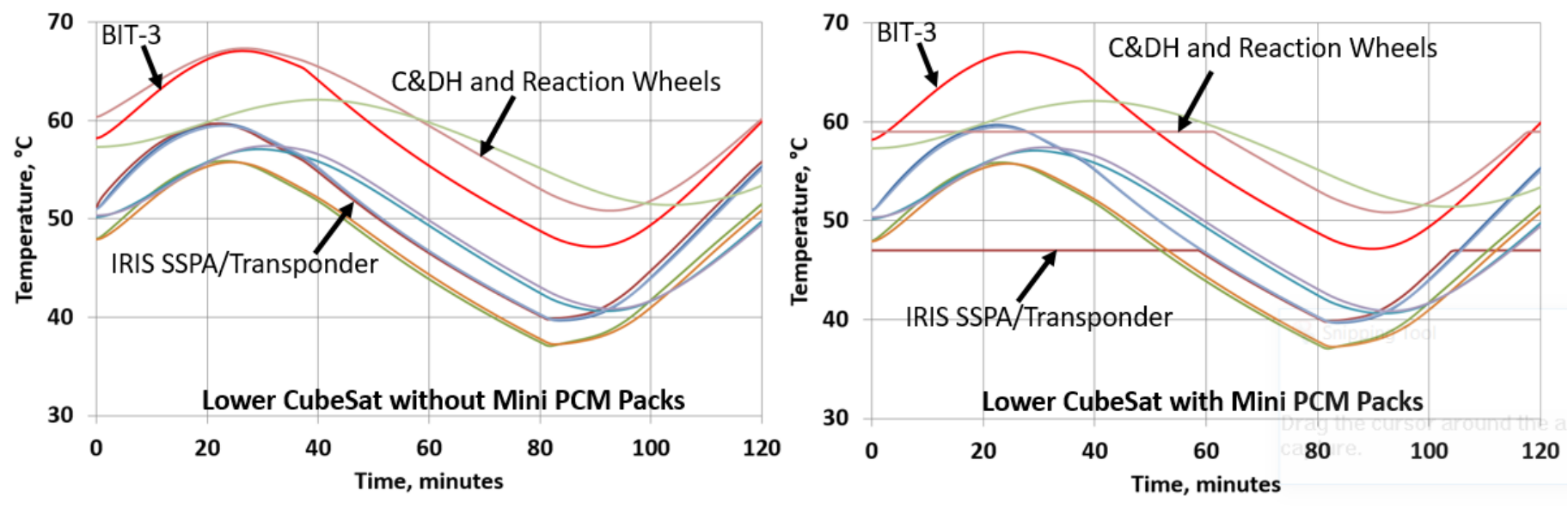

Fig. 13. Hot case temperature predictions in lunar orbit at $90 \mathrm{~km}$ altitude above lunar surface during spiraling down from ESPA to Moon.

Figure 14 presents the worst hot case temperature predictions for the BOLAS lower altitude CubeSat in an elliptical lunar orbit with a true perigee height of $2.01 \mathrm{~km}$ and an eccentricity of 0.039184 . For this orbit, the instruments are powered on and the BIT-3s are powered off. All the temperatures are within the allowable flight temperature limits. The temperatures of the C\&DH, Iris SSPA, and transponder are below the melting points of the PCMs. No PCM phase change occurs. 


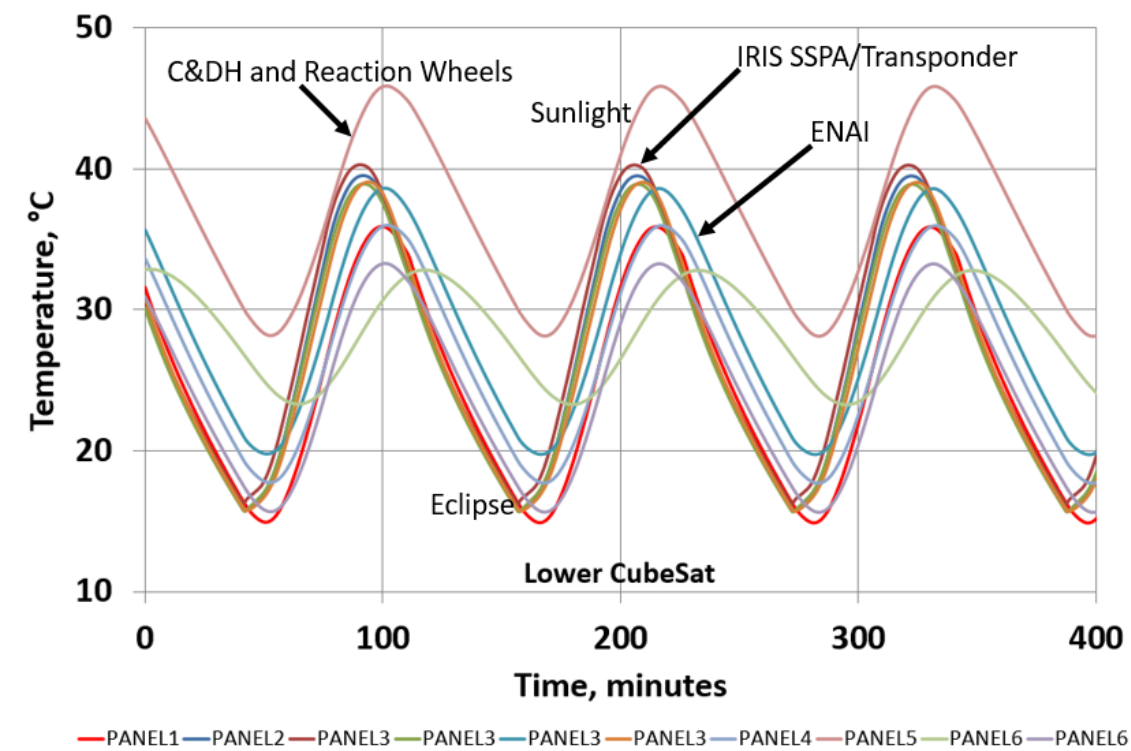

Fig. 14. Hot case temperature predictions for elliptical lunar orbit (true perigee height=2.01 km; eccentricity $=\mathbf{0 . 0 3 9 1 8 4}$ ) with payload on and BIT-3s off.

Figure 15 presents the worst hot case temperature predictions for the BOLAS lower altitude CubeSat during BIT-3 thrusting to change the frozen orbit altitude above the lunar surface from $90 \mathrm{~km}$ to $14.51 \mathrm{~km}$. With PCM, all the temperatures are within the allowable flight temperature limits.

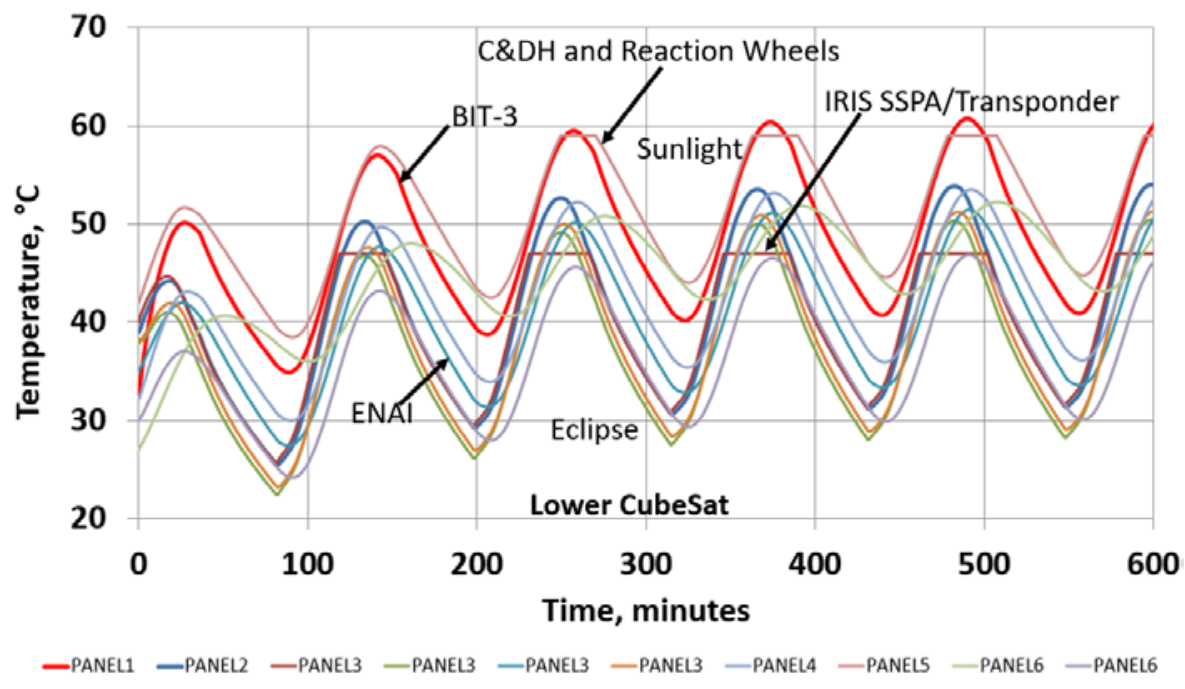

Fig. 15. Hot case temperature predictions during BIT-3 thrusting to change orbit altitude from $90 \mathrm{~km}$ to $14.51 \mathrm{~km}$.

\section{Paraffin PCM Flight Heritage}

Paraffin PCM has NASA flight heritage, including IceCube, NICER, and MESSENGER. ${ }^{5}$ Figure 16 shows the IceCube flight miniature paraffin PCM packs. 


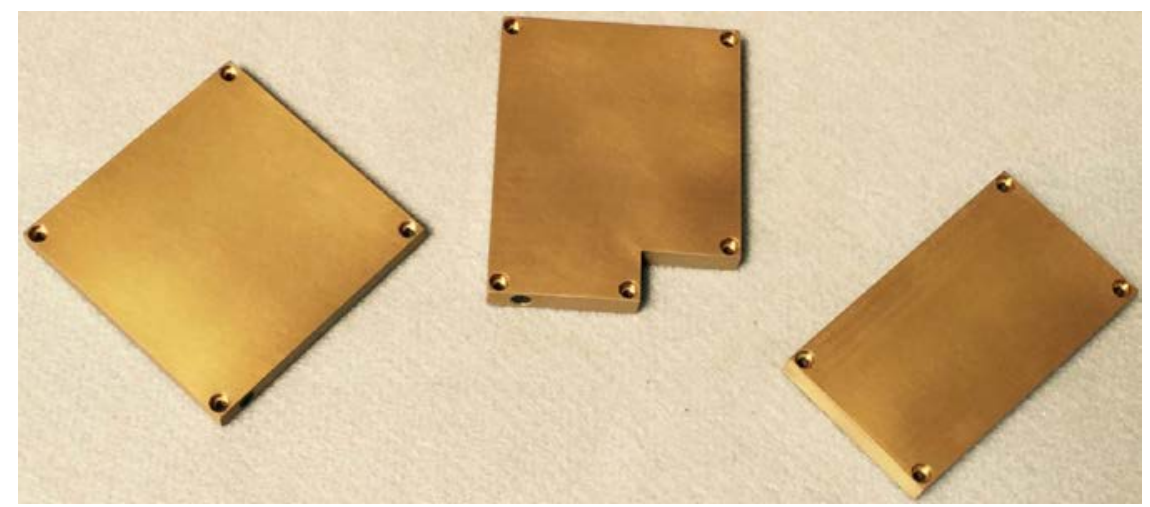

Fig. 16. IceCube flight miniature paraffin PCM packs.

\section{Conclusion}

BOLAS is a NASA planetary CubeSat mission concept. The thermal management approach uses passive or radiative cooling and paraffin PCM to maintain the bi-sat component temperatures within the maximum operating limits in low Lunar orbit. The exterior of the CubeSat chassis is used as radiators. The BOLAS lower CubeSat is at a $90 \mathrm{~km}$ altitude above the lunar surface during spiraling down from the ESPA to the Moon. Without PCM, the worst hot case temperature predictions for the C\&DH exceeds the $61^{\circ} \mathrm{C}$ maximum operating limit, and the Iris SSPA and transponder temperatures exceed the $50^{\circ} \mathrm{C}$ maximum operating limit. Miniature paraffin PCM packs are used to store thermal energy in sunlight and release it in the eclipse. The PCM on the Iris SSPA and transponder is nTricosane $\left(\mathrm{C}_{23} \mathrm{H}_{48}\right)$, which has a $47^{\circ} \mathrm{C}$ melting point and $166 \mathrm{~kJ} / \mathrm{kg}$ enthalpy of fusion. The mass of miniature $\mathrm{n}$ Tricosane PCM packs, including the aluminum structure, is $220 \mathrm{~g}$ per CubeSat. The PCM on the C\&DH is nHexacosane $\left(\mathrm{C}_{27} \mathrm{H}_{56}\right)$, which has a $57^{\circ} \mathrm{C}$ melting point and $162 \mathrm{~kJ} / \mathrm{kg}$ enthalpy of fusion. The mass of miniature nHexacosane PCM packs, including the aluminum structure, is $50 \mathrm{~g}$ per CubeSat. With paraffin PCM, all the temperatures are within the maximum operating limits. The miniature paraffin PCM packs are attached to the C\&DH, Iris SSPA, and transponder. Thermal interface materials, such as indium, are used to maximize the interface conductances at the attachment joints.

\section{Acknowledgments}

This material is based upon work supported by NASA Planetary Science Deep Space SmallSat Studies (PSDS3) program. The author thanks the following individuals for their technical contributions: Timothy Stubbs (BOLAS Principal Investigator) of GSFC Code 695; Michael Mesarch of GSFC Code 595; Benjamin Malphrus of Morehead State University).

\section{References}

[1] Stubbs, T. J., et al., "Bi-sat Observations of the Lunar environment Above Swirls (BOLAS): Tethered Microsat Investigation of Space Weathering and the Water Cycle at the Moon", 49 ${ }^{\text {th }}$ Lunar and Planetary Science Conference 2018 (LPI Contrib. No. 2083).

[2] Baker, C., et al., "Lunar Reconnaissance Orbiter (LRO) Thermal On-Orbit Performance“, AIAA 2011-5097, 41 ${ }^{\text {st }}$ International Conference on Environmental Systems, 17 - 21 July 2011, Portland, OR.

[3] Gerhardt, D., et al., "The Colorado Student Space Weather Experiment (CSSWE) On-Orbit Performance”, Swartwout, M. (2013): JoSS, Vol. 3, No. 1, pp. 265-281.

[4] Clark, C. S., et al., "Power System Challenges for Small Satellite Missions”, Clyde Space Ltd., 2006 European Small Satellite Services Symposium.

[5] Choi, M. K., “Thermal Assessment of Paraffin Phase Change Material Mini-Packs on IceCube 3U CubeSat in Flight”, AIAA paper 2018-4490, 2018 AIAA Propulsion and Energy Forum, Cincinnati, OH. 